\title{
Polynomials associated with an algebraic solution of the sixth Painlevé equation
}

\author{
By Makoto TANedA \\ (Received June 16, 1999) \\ (Revised April 13, 2000) \\ (from Nagoya Mathematical Journal)
}

\section{Introduction}

For a non-negative integer $n$, let $T_{n}$ be the rational function determined by the recurrence relation

$$
\begin{aligned}
T_{n-1} T_{n+1}= & \left\{\frac{1}{4}\left(-2 b_{1}^{2}-2 b_{2}^{2}+\left(b_{1}^{2}-b_{2}^{2}\right) v\right)+\left(n-\frac{1}{2}\right)^{2}\right\} T_{n}^{2} \\
& +\frac{1}{4}\left(v^{2}-4\right)^{2}\left\{T_{n} \frac{d^{2} T_{n}}{d v^{2}}-\left(\frac{d T_{n}}{d v}\right)^{2}\right\} \\
& +\frac{1}{4}\left(v^{2}-4\right) v T_{n} \frac{d T_{n}}{d v}
\end{aligned}
$$

with initial conditions $T_{0}=T_{1}=1$, where $v$ is a variable and $b_{1}$ and $b_{2}$ are parameters. If you enjoy calculating a first few $T_{n}$ 's by Mathematica, you will realize that they are polynomials. The polynomials $T_{n}$ are, however quite complicated ([1]). A transformation given in [2] yields a surprising simplification. Namely, we put $z=(2-v) / 4, w=(2+v) / 4, c=-4 b_{1}^{2}$ and $d=-4 b_{2}^{2}$. For a positive integer $k$, we denote $\overline{c_{k}}=\left(c+(2 k-1)^{2}\right), \overline{d_{k}}=\left(d+(2 k-1)^{2}\right)$ and define $c_{k}=\overline{c_{1}} \overline{c_{2}} \overline{c_{3}} \cdots \overline{c_{k}}$, $d_{k}=\overline{d_{1}} \overline{d_{2}} \overline{d_{3}} \cdots \overline{d_{k}}$. See the formulae (9) and (10) below.

We denote, for a positive integer $n$, by $[n]$ the set $[n]=\{1,2,3, \ldots, n\}$. For a subset $I=\left\{i_{1}, i_{2}, \ldots, i_{p}\right\} \subset[n-1]$, we define the weight $\|I\|$ by

$$
\|I\|=i_{1}+i_{2}+\cdots+i_{p}
$$

We put $c_{\emptyset}=1$ and $d_{\emptyset}=1$. For a subset $I=\left\{i_{1}, i_{2}, \ldots, i_{p}\right\} \subset[n-1], I \neq \emptyset$, we set $c_{I}=c_{i_{1}} c_{i_{2}} \cdots c_{i_{p}}$ and $d_{I}=d_{i_{1}} d_{i_{2}} \cdots d_{i_{p}}$. For a partition $\lambda=\left(\lambda_{1} \geq \lambda_{2} \geq \cdots \geq \lambda_{q}\right)$, we define $p(\lambda)=\#\left\{i \in \mathbf{Z}: 1 \leq i \leq q, \lambda_{i} \geq i\right\}, \lambda_{i}^{\prime}=\#\left\{j \in \mathbf{Z}: 1 \leq j \leq q, \lambda_{j} \geq i\right\}$, $\alpha_{i}=\lambda_{i}-i$ and $\beta_{i}=\lambda_{i}^{\prime}-i$ for $1 \leq i \leq p(\lambda)$. See the example below. Since $\alpha_{i}, \beta_{i}$, 
$1 \leq i \leq p(\lambda)$ determine the partition we write

$$
\lambda=\left(\alpha_{1}, \alpha_{2}, \ldots, \alpha_{p(\lambda)} \mid \beta_{1}, \beta_{2}, \ldots, \beta_{p(\lambda)}\right),
$$

which is called the Frobenius notation. For a partition $\lambda=\left(\lambda_{1} \geq \lambda_{2} \geq \cdots \geq \lambda_{q}\right)$, we define the Young diagram $\bar{\lambda}$ by

$$
\bar{\lambda}=\left\{(i, j) \in Z \times Z: 1 \leq j \leq \lambda_{i}, 1 \leq i \leq q\right\} .
$$

The Young diagram is often illustrated with squares called celles. For a cell $x=$ $(i, j) \in \bar{\lambda}$, we define its content $c(x)$ and hook-length $h(x)$ by

$$
\begin{aligned}
& c(x)=j-i, \\
& h(x)=\lambda_{i}+\lambda_{j}^{\prime}-i-j+1 .
\end{aligned}
$$

As this example shows for a partition $\lambda=(5 \geq 4 \geq 2 \geq 1)$ for which $\lambda_{1}=5$, $\lambda_{2}=4, \lambda_{3}=2, \lambda_{4}=1$, we have $\lambda_{1}^{\prime}=4, \lambda_{2}^{\prime}=3, \lambda_{3}^{\prime}=2, \lambda_{4}^{\prime}=2, \lambda_{5}^{\prime}=1$, $p(\lambda)=2, \alpha_{1}=4, \alpha_{2}=2, \beta_{1}=3, \beta_{2}=1$ and hence the Frobenius notation of $\lambda$ is $\lambda=(4,2 \mid 3,1)$. The Young diagram of $\bar{\lambda}$ is
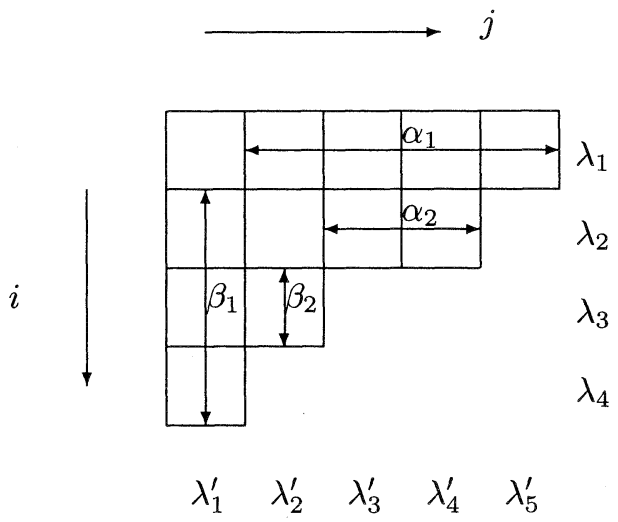

Figure

so that $\lambda_{i}$ is the number of cells in the $i$-th row and $\lambda_{i}^{\prime}$ is the number of cells in the $i$-th column.

We associate to a strictly decreasing partition $I=\left\{i_{1}>i_{2}>\cdots>i_{p}\right\} \neq \emptyset$ a partition

$$
\lambda(I)=\left(i_{1}-1, i_{2}-1, \ldots, i_{p}-1 \mid i_{1}, i_{2}, \ldots, i_{p}\right)
$$


It is convenient to put $\lambda(\emptyset)=\emptyset, \prod_{x \in \emptyset} f(x)=1$ and $\sum_{x \in \emptyset} f(x)=0$. With this notation, we can state Conjecture (7.3) in [2].

Theorem 1. For an integer $n \geq 2$, we have

$$
T_{n}=2^{-n(n-1)} \sum_{I \subset[n-1]}\left\{\prod_{x \in \overline{\lambda(I)}}\left(\frac{n+c(x)}{h(x)}\right) c_{I} d_{[n-1]-I} z^{\|I\|} w^{\|[n-1]-I\|}\right\}
$$

The purpose of this paper is to prove Theorem 1.

For example, Theorem 1 tells us

$$
\begin{aligned}
& T_{2}=2^{-1}\left(c_{1} z+d_{1} w\right) \\
& T_{3}=2^{-6}\left(c_{1} c_{2} z^{3}+3 d_{1} c_{2} z^{2} w+3 c_{1} d_{2} z w^{2}+d_{1} d_{2} w^{3}\right) .
\end{aligned}
$$

\section{$\S 1$. Some Propositions}

For an integer $n \geq 2$ and a subset $I \subset[n-1]$, we set

$$
\mu_{n}(I)=\prod_{x \in \overline{\lambda(I)}} \frac{n+c(x)}{h(x)}
$$

We notice here that $\mu_{n}(I)$ is the dimension of the irreducible representation of $G L_{n}$ associated to partition $\lambda(I)$. So Theorem 1 reveals a new aspect of Painlevé equations, an unexpected relation between combinatorics of the symmetric groups and the Painlevé equations. We first prove the following two Propositions.

Proposition 1. For an integer $n \geq 2$ and a subset $I \in[n-1]$, we have

$$
\mu_{n}(I)=\prod_{\nu \in I}\left(\frac{n-\nu}{n+\nu}\right) \mu_{n+1}(I) .
$$

Proof. We put $I=\left\{i_{1}>i_{2}>\cdots>i_{p}\right\} \subset[n-1]$ and denote $\overline{\lambda_{k}(I)}=$ $\{(i, j) \in \overline{\lambda(I)}: i=k, j \geq k$ or $j=k, i \geq k\}$. Then we have

$$
\begin{aligned}
\prod_{x \in \overline{\lambda(I)}}(n+c(x)) & =\prod_{k=1}^{p}\left\{\prod_{x \in \overline{\lambda_{k}(I)}}(n+c(x))\right\} \\
& =\prod_{k=1}^{p}\left\{\prod_{j=0}^{2 i_{k}-1}\left(n-i_{k}+j\right)\right\}
\end{aligned}
$$




$$
\begin{aligned}
& =\prod_{k=1}^{p}\left\{\left(\frac{n-i_{k}}{n+i_{k}}\right) \prod_{x \in \overline{\lambda_{k}(I)}}(n+1+c(x))\right\} \\
& =\prod_{\nu \in I}\left(\frac{n-\nu}{n+\nu}\right) \prod_{x \in \overline{\lambda(I)}}(n+1+c(x)) .
\end{aligned}
$$

So the denominator of $\mu_{n}(I)$ is equal to that of $\mu_{n+1}(I)$, and hence Proposition 1 is proved.

Proposition 2. For an integer $n \geq 2$ and a subset $I \subset[n-1]$, we have

$$
\mu_{n}(I)=\mu_{n}([n-1]-I)
$$

Proof. We prove Proposition 2 by induction on $n$. As we have $\mu_{2}(\emptyset)=1$ and $\mu_{2}(\{1\})=1$, Proposition 2 holds for $n=2$. Suppose that Proposition 2 is verified for $n=N-1, N \geq 3$. We put $I=\left\{i_{1}>i_{2}>\cdots>i_{p}\right\} \subset[N-1]$ so that $\lambda_{1} \neq N-1$. It follows from Proposition 1 that

$$
\begin{aligned}
\mu_{N}([N-1]-I) \\
\quad=\prod_{\nu \in[N-1]-I}\left(\frac{N-1+\nu}{N-1-\nu}\right) \mu_{N-1}([N-1]-I) \\
\quad=\prod_{\nu \in[N-1]-I}\left(\frac{N-1+\nu}{N-1-\nu}\right) \mu_{N-1}(I-\{N-1\}) \\
\quad=\prod_{\nu \in[N-1]-I}\left(\frac{N-1+\nu}{N-1-\nu}\right) \prod_{\nu \in I-\{N-1\}}\left(\frac{N-1-\nu}{N-1+\nu}\right) \mu_{N}(I-\{N-1\}) .
\end{aligned}
$$

Now we can see that the content and the hook-length of a cell $(i, j) \in \overline{\lambda(I)}, i \geq$ $2, j \geq 2$ is equal to the content and the hook-length of a cell $(i-1, j-1) \in$ $\overline{\lambda(I-\{N-1\})}$. Moreover we have

$$
\begin{aligned}
& \prod_{x \in \overline{\lambda_{1}(I)}}(N+c(x))=(2 N-2) !, \\
& h((1,1))=2 N-2, \quad h((p+1,1))=N-1, \\
& h((k, 1))=h((1, k))=N-1+i_{k}, \quad(2 \leq k \leq p) .
\end{aligned}
$$

We now put $[N-1]-I=\left\{j_{1}>j_{2}>\cdots>j_{N-1-p}\right\}$. Then we have

$$
h((N+1-k, 1))=h((1, N-k))=N-1-j_{k}, \quad(1 \leq k \leq N-1-p) .
$$


Therefore, we have

$$
\begin{aligned}
& \mu_{N}(I-\{N-1\}) \\
& =\frac{1}{(2 N-2) !}(2 N-2)(N-1)\left\{\prod_{\nu \in[N-1]-I}(N-1-\nu)\right\}^{2} \\
& \quad \times\left\{\prod_{\nu \in I-\{N-1\}}(N-1+\nu)\right\}^{2} \mu_{N}(I) .
\end{aligned}
$$

Combining the identity (20) with the identity (15), we have

$$
\mu_{N}([N-1]-I)=\mu_{N}(I),
$$

which is equality (12) for $n=N$. Hence we have completed the mathematical induction and verified Proposition 2.

Now we need the following

Definition 1. For an integer $n \geq 2$, subsets $I, J \in[n-1]$, a positive integer $\lambda \in I \cup J$, we define $b_{\lambda}^{I, J}$ by the following partial fractional decomposition

$$
\begin{aligned}
& \prod_{\lambda \in I}\left(\frac{x+\lambda}{x-\lambda}\right) \prod_{\lambda \in J}\left(\frac{x-1-\lambda}{x-1+\lambda}\right)+\prod_{\lambda \in I}\left(\frac{x-1-\lambda}{x-1+\lambda}\right) \prod_{\lambda \in J}\left(\frac{x+\lambda}{x-\lambda}\right) \\
& \quad=2+\sum_{\lambda \in I \cup J} \frac{b_{\lambda}^{I, J}}{(x-\lambda)(x-1+\lambda)} .
\end{aligned}
$$

In particular, for an element $\lambda \in I \cap J$, we have

$$
\begin{aligned}
b_{\lambda}^{I, J}=2 \lambda(2 \lambda-1)\left\{\prod_{\nu \in I-\{\lambda\}}\right. & \left(\frac{\lambda+\nu}{\lambda-\nu}\right) \prod_{\nu \in J}\left(\frac{\lambda-1-\nu}{\lambda-1+\nu}\right) \\
& \left.+\prod_{\nu \in I}\left(\frac{\lambda-1-\nu}{\lambda-1+\nu}\right) \prod_{\nu \in J-\{\lambda\}}\left(\frac{\lambda+\nu}{\lambda-\nu}\right)\right\} .
\end{aligned}
$$

For an element $\lambda \in I-I \cup J$, we have

$$
b_{\lambda}^{I, J}=2 \lambda(2 \lambda-1) \prod_{\nu \in I-\{\lambda\}}\left(\frac{\lambda+\nu}{\lambda-\nu}\right) \prod_{\nu \in J}\left(\frac{\lambda-1-\nu}{\lambda-1+\nu}\right) .
$$

The following two Lemmas are easy consequences of Definition 1 . The union $A \cup B$ of two subsets $A, B$ of a set $S$ will be sometimes denoted by $A+B$. 
Lemma 1. We have

$$
\sum_{\lambda \in I \cup J} b_{\lambda}^{I, J}=4(\|I\|-\|J\|)^{2}-2(\|I\|+\|J\|)
$$

Lemma 2. For an element $\lambda \in I \cap J$, we have $b_{\lambda}^{I, J}=0$ if and only if $\lambda-1 \epsilon$ $I \cap J$. For an element $\lambda \in I-I \cap J$, we have $b_{\lambda}^{I, J}=0$ if and only if $\lambda-1 \in J$.

To prove Theorem 1 , we need also the following

Definition 2. For an integer $n \geq 2, I, J \subset[n-1]$, an integer $\lambda \in I \cup J$, we define $F P(I, J)$ by

$$
F P(I, J)=\mu_{n}(I) \mu_{n}(J)\left(1+\sum_{\lambda \in I \cup J} \frac{b_{\lambda}^{I, J}}{2(n-\lambda)(n-1+\lambda)}\right)
$$

and $S P(n, \lambda, I, J)$ by

$$
S P(n, \lambda, I, J)=b_{\lambda}^{I, J} \mu_{n}(I) \mu_{n}(J) .
$$

We set

$$
\begin{aligned}
& T P(n, 1, I+\{1\} ; J+\{1\}) \\
& \quad=-2 \prod_{\nu \in I}\left(\frac{1+\nu}{1-\nu}\right)(-1)^{\|J\|} \mu_{n}(I+\{1\}) \mu_{n}(J+\{1\})
\end{aligned}
$$

for an integer $n \geq 2$ and subsets $I, J \subset[n-1]-\{1\}$, and

$$
\begin{aligned}
T P(n, \lambda, I+\{\lambda\} ; J+\{\lambda\}) & \\
= & 2 \lambda(2 \lambda-1) \prod_{\nu \in I}\left(\frac{\lambda+\nu}{\lambda-\nu}\right) \prod_{\nu \in J+\{\lambda\}}\left(\frac{\lambda-1-\nu}{\lambda-1+\nu}\right) \\
& \times \mu_{n}(I+\{\lambda\}) \mu_{n}(J+\{\lambda\})
\end{aligned}
$$

for an integer $n \geq 3$, an integer $2 \leq \lambda \leq n-1$ and subsets $I, J \subset[n-1]-\{\lambda\}-$ $\{\lambda-1\}$.

We can see $F P(I, J)=F P(J, I)$ and $S P(n, \lambda, I, J)=S P(n, \lambda, J, I)$. From Proposition 1 and Definitions 1,2 , we immediately obtain the following 
Proposition 3. For an integer $n \geq 2$, we have

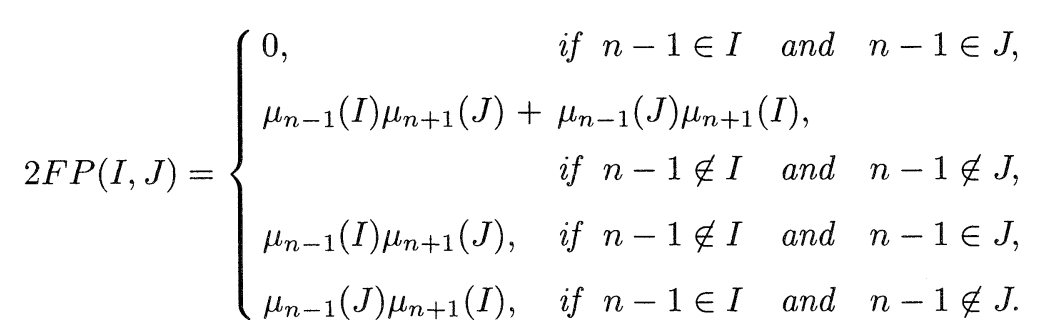

We now prove the following three lemmas.

LEMMA 3. For an integer $n \geq 2$ and subsets $I, I^{\prime}, J, J^{\prime} \subset[n-1]-\{1\}$ such that $I+I^{\prime}=[n-1]-\{1\}, J+J^{\prime}=[n-1]-\{1\}$, we have

$$
T P(n, 1, I+\{1\} ; J+\{1\})+S P\left(n, 1, I^{\prime}+\{1\}, J^{\prime}\right)=0 .
$$

Proof. We prove Lemma 3 by mathematical induction on $n$. As we have $T P(2,1,\{1\} ;\{1\})=-2, S P(2,1,\{1\}, \emptyset)=2$. Lemma 3 holds for $n=2$. Suppose that Lemma 3 is proved for $n=N-1, N \geq 3$. We shall prove Lemma 3 for $n=N$. Let $H P(N, 1, I, J)$ be the function defined by

$$
H P(N, 1, I, J)=\left\{\begin{aligned}
\prod_{\nu \in I+\{1\}}\left(\frac{N-1+\nu}{N-1-\nu}\right) \prod_{\nu \in I+\{1\}}\left(\frac{N-1+\nu}{N-1-\nu}\right), & \text { if } N-1 \notin I, N-1 \notin J, \\
-\prod_{\nu \in I^{\prime}+\{1\}}\left(\frac{N-1+\nu}{N-1-\nu}\right) \prod_{\nu \in J+\{1\}}\left(\frac{N-1+\nu}{N-1-\nu}\right), & \text { if } N-1 \in I, N-1 \notin J, \\
-\prod_{\nu \in I+\{1\}}\left(\frac{N-1+\nu}{N-1-\nu}\right) \prod_{\nu \in J^{\prime}}\left(\frac{N-1+\nu}{N-1-\nu}\right), & \text { if } N-1 \notin I, N-1 \in J, \\
\prod_{\nu \in I^{\prime}+\{1\}}\left(\frac{N-1+\nu}{N-1-\nu}\right) \prod_{\nu \in J^{\prime}}\left(\frac{N-1+\nu}{N-1-\nu}\right), & \text { if } N-1 \in I, N-1 \in J .
\end{aligned}\right.
$$

By Propositions 1, 2 and Definitions 1, 2, we have

$$
\begin{aligned}
& T P(N, 1, I+\{1\} ; J+\{1\}) \\
& \quad=T P(N-1,1, I+\{1\} ; J+\{1\}) H P(N, 1, I, J),
\end{aligned}
$$




$$
\begin{aligned}
& S P\left(N, 1, I^{\prime}+\{1\}, J^{\prime}\right) \\
& \quad=S P\left(N-1,1, I^{\prime}+\{1\}-\{N-1\}, J^{\prime}-\{N-1\}\right) H P(N, 1, I, J) .
\end{aligned}
$$

By the induction hypothesis, we have

$$
\begin{aligned}
& T P(N, 1, I+\{1\} ; J+\{1\})+S P\left(N, 1, I^{\prime}+\{1\}, J^{\prime}\right) \\
& =H P(N, 1, I, J)\{T P(N-1,1, I+\{1\} ; J+\{1\}) \\
& \left.\quad+S P\left(N-1,1, I^{\prime}+\{1\}-\{N-1\}, J^{\prime}-\{N-1\}\right)\right\} \\
& =0 .
\end{aligned}
$$

We have verified Lemma 3 for $n=N$. Hence Lemma 3 is proved by mathematical induction on $n$.

Lemma 4. For an integer $n \geq 3$, an integer $2 \leq \lambda \leq n-1$ and subsets $I, I^{\prime}, J, J^{\prime} \subset[n-1]-\{\lambda\}-\{\lambda-1\}$ such that $I+I^{\prime}=[n-1]-\{\lambda\}-\{\lambda-1\}$, $J+J^{\prime}=[n-1]-\{\lambda\}-\{\lambda-1\}$, we have

$$
T P(n, \lambda, I+\{\lambda\} ; J+\{\lambda\})+S P\left(n, 1, I^{\prime}+\{\lambda-1\}+\{\lambda\}, J^{\prime}\right)=0
$$

Proof. We prove Lemma 4 by mathematical induction on $n$. As we have $T P(3,2,\{2\} ;\{2\})=-36$ and $S P(3,2,\{1,2\}, \emptyset)=36$, Lemma 4 holds for $n=3$. Suppose that Lemma 4 is proved for $n=N-1, N \geq 4$. First, we shall prove Lemma 4 for $n=N, \lambda \neq N-1$. Let $\operatorname{HP}(N, \lambda, I, J)$ be the function defined by

$$
H P(N, \lambda, I, J)=\left\{\begin{array}{r}
\prod_{\nu \in I+\{\lambda\}}\left(\frac{N-1+\nu}{N-1-\nu}\right) \prod_{\nu \in J+\{\lambda\}}\left(\frac{N-1+\nu}{N-1-\nu}\right), \\
\text { if } N-1 \notin I, N-1 \notin J, \\
-\prod_{\nu \in I^{\prime}+\{\lambda-1\}+\{\lambda\}}\left(\frac{N-1+\nu}{N-1-\nu}\right) \prod_{\nu \in J+\{\lambda\}}\left(\frac{N-1+\nu}{N-1-\nu}\right), \\
\quad \text { if } N-1 \in I, N-1 \notin J, \\
-\prod_{\nu \in I+\{\lambda\}}\left(\frac{N-1+\nu}{N-1-\nu}\right) \prod_{\nu \in J^{\prime}}\left(\frac{N-1+\nu}{N-1-\nu}\right), \\
\quad \text { if } N-1 \notin I, N-1 \in J, \\
\prod_{\nu \in I^{\prime}+\{\lambda-1\}+\{\lambda\}}\left(\frac{N-1+\nu}{N-1-\nu}\right) \prod_{\nu \in J^{\prime}}\left(\frac{N-1+\nu}{N-1-\nu}\right), \\
\quad \text { if } N-1 \in I, N-1 \in J .
\end{array}\right.
$$


By Propositions 1, 2 and Definitions 1, 2, we have

$$
\begin{aligned}
& T P(N, \lambda, I+\{\lambda\} ; J+\{\lambda\}) \\
&= T P(N-1, \lambda, I+\{\lambda\} ; J+\{\lambda\}) H P(N, \lambda, I, J), \\
& S P\left(N, 1, I^{\prime}+\{\lambda-1\}+\{\lambda\}, J^{\prime}\right) \\
&= S P\left(N-1,1, I^{\prime}+\{\lambda-1\}+\{\lambda\}-\{N-1\}, J^{\prime}-\{N-1\}\right) \\
& \times H P(N, \lambda, I, J) .
\end{aligned}
$$

By the induction hypothesis, we have

$$
\begin{aligned}
& T P(N, \lambda, I+\{\lambda\} ; J+\{\lambda\})+S P\left(N, \lambda, I^{\prime}+\{\lambda-1\}+\{\lambda\}, J^{\prime}\right) \\
& =H P(N, \lambda, I, J) \\
& \quad \times\{T P(N-1, \lambda, I+\{\lambda\} ; J+\{\lambda\}) \\
& \left.\quad+S P\left(N-1, \lambda, I^{\prime}+\{\lambda-1\}+\{\lambda\}-\{N-1\}, J^{\prime}-\{N-1\}\right)\right\} \\
& =0 .
\end{aligned}
$$

Hence, we have verified Lemma 4 for $n=N, \lambda \neq N-1$.

It remains to prove Lemma 4 for $n=N, \lambda=N-1$. By Propositions 1,2 and Definitions 1, 2, we have

$$
\begin{aligned}
T P(N, & N-1, I+\{N-1\} ; J+\{N-1\}) \\
= & -2(N-1) \prod_{\nu \in I}\left(\frac{N-1+\nu}{N-1-\nu}\right) \prod_{\nu \in J}\left(\frac{N-2-\nu}{N-2+\nu}\right) \\
& \times \mu_{N}(I+\{N-1\}) \mu_{N}(J+\{N-1\}) .
\end{aligned}
$$

Now, we shall prove

$$
\begin{aligned}
& \mu_{N}\left(I^{\prime}+\{N-2\}+\{N-1\}\right) \\
& =\prod_{\nu \in I}\left(\frac{N-1+\nu}{N-1-\nu}\right) \prod_{\nu \in I^{\prime}+\{N-2\}}\left(\frac{N-1-\nu}{N-1+\nu}\right) \mu_{N}(I+\{N-1\}) .
\end{aligned}
$$

It follows from Proposition 2 that

$$
\mu_{N}\left(I^{\prime}+\{N-2\}+\{N-1\}\right)=\mu_{N}(I)
$$

by Proposition 1 for $n=N-2$,

$$
=\prod_{\nu \in I}\left(\frac{N-1+\nu}{N-1-\nu}\right) \mu_{N-1}(I)
$$


by Proposition 2,

$$
=\prod_{\nu \in I}\left(\frac{N-1+\nu}{N-1-\nu}\right) \mu_{N-1}\left(I^{\prime}+\{N-2\}\right)
$$

by Proposition 1 for $n=N-1$,

$$
=\prod_{\nu \in I}\left(\frac{N-1+\nu}{N-1-\nu}\right) \prod_{\nu \in I^{\prime}+\{N-2\}}\left(\frac{N-1-\nu}{N-1+\nu}\right) \mu_{N}\left(I^{\prime}+\{N-2\}\right)
$$

by Proposition 2,

$$
=\prod_{\nu \in I}\left(\frac{N-1+\nu}{N-1-\nu}\right) \prod_{\nu \in I^{\prime}+\{N-2\}}\left(\frac{N-1-\nu}{N-1+\nu}\right) \mu_{N}(I+\{N-1\}) .
$$

We hence have the identity (42).

Now, we shall prove

$$
\begin{aligned}
\mu_{N}\left(J^{\prime}\right)= & \prod_{\nu \in J^{\prime}}\left(\frac{N-1+\nu}{N-1-\nu}\right) \prod_{\nu \in J^{\prime}}\left(\frac{N-2+\nu}{N-2-\nu}\right) \prod_{\nu \in J}\left(\frac{N-2-\nu}{N-2+\nu}\right) \\
& \times \prod_{\nu \in J^{\prime}+\{N-2\}}\left(\frac{N-1-\nu}{N-1+\nu}\right) \mu_{N}(J+\{N-1\}) .
\end{aligned}
$$

We apply Proposition 1 for $n=N-2$ and $n=N-1$ to get

$$
\mu_{N}\left(J^{\prime}\right)=\prod_{\nu \in J^{\prime}}\left(\frac{N-1+\nu}{N-1-\nu}\right) \prod_{\nu \in J^{\prime}}\left(\frac{N-2+\nu}{N-2-\nu}\right) \mu_{N-2}\left(J^{\prime}\right)
$$

by Proposition 2,

$$
=\prod_{\nu \in J^{\prime}}\left(\frac{N-1+\nu}{N-1-\nu}\right) \prod_{\nu \in J^{\prime}}\left(\frac{N-2+\nu}{N-2-\nu}\right) \mu_{N-2}(J)
$$

by Proposition 1 for $n=N-2$,

$$
=\prod_{\nu \in J^{\prime}}\left(\frac{N-1+\nu}{N-1-\nu}\right) \prod_{\nu \in J^{\prime}}\left(\frac{N-2+\nu}{N-2-\nu}\right) \prod_{\nu \in J}\left(\frac{N-2-\nu}{N-2+\nu}\right) \mu_{N-1}(J)
$$

by Proposition 2,

$$
=\prod_{\nu \in J^{\prime}}\left(\frac{N-1+\nu}{N-1-\nu}\right) \prod_{\nu \in J^{\prime}}\left(\frac{N-2+\nu}{N-2-\nu}\right) \prod_{\nu \in J}\left(\frac{N-2-\nu}{N-2+\nu}\right) \mu_{N-1}\left(J^{\prime}+\{N-2\}\right)
$$


by Proposition 1 for $n=N-1$,

$$
\begin{aligned}
= & \prod_{\nu \in J^{\prime}}\left(\frac{N-1+\nu}{N-1-\nu}\right) \prod_{\nu \in J^{\prime}}\left(\frac{N-2+\nu}{N-2-\nu}\right) \prod_{\nu \in J}\left(\frac{N-2-\nu}{N-2+\nu}\right) \\
& \times \prod_{\nu \in J^{\prime}+\{N-2\}}\left(\frac{N-1-\nu}{N-1+\nu}\right) \mu_{N}\left(J^{\prime}+\{N-2\}\right)
\end{aligned}
$$

by Proposition 2,

$$
\begin{aligned}
= & \prod_{\nu \in J^{\prime}}\left(\frac{N-1+\nu}{N-1-\nu}\right) \prod_{\nu \in J^{\prime}}\left(\frac{N-2+\nu}{N-2-\nu}\right) \prod_{\nu \in J}\left(\frac{N-2-\nu}{N-2+\nu}\right) \\
& \times \prod_{\nu \in J^{\prime}+\{N-2\}}\left(\frac{N-1-\nu}{N-1+\nu}\right) \mu_{N}(J+\{N-1\}) .
\end{aligned}
$$

We hence have the identity (44).

By the identities (42), (44) and Definitions 1, 2, we get

$$
\begin{aligned}
S P(N, & \left.N-1, I^{\prime}+\{N-2\}+\{N-1\}, J^{\prime}\right) \\
= & 2(N-1) \prod_{\nu \in I}\left(\frac{N-1+\nu}{N-1-\nu}\right) \prod_{\nu \in J}\left(\frac{N-2-\nu}{N-2+\nu}\right) \\
& \times \mu_{N}(I+\{N-1\}) \mu_{N}(J+\{N-1\}) .
\end{aligned}
$$

Combining the identity (46) with the identity (41), we have

$$
\begin{aligned}
& T P(N, N-1, I+\{N-1\} ; J+\{N-1\}) \\
& \quad+S P\left(N, N-1, I^{\prime}+\{N-2\}+\{N-1\}, J^{\prime}\right)=0 .
\end{aligned}
$$

So, Lemma 4 holds for $n=N, \lambda=N-1$. We have verified Lemma 4 for $n=N$ and Lemma 4 is proved by mathematical induction.

Lemma 5. For an integer $n \geq 3$, an integer $2 \leq \lambda \leq n-1$ and subsets $I, I^{\prime}$, $J, J^{\prime}$ such that $I+I^{\prime}=[n-1]-\{\lambda\}-\{\lambda-1\}, J+J^{\prime}=[n-1]-\{\lambda\}-\{\lambda-1\}$, we have

$$
S P(n, \lambda, I+\{\lambda-1\}+\{\lambda\}, J+\{\lambda\})+S P\left(n, \lambda, I^{\prime}+\{\lambda\}, J^{\prime}\right)=0
$$

Proof. We prove Lemma 5 as well as Lemma 4 by mathematical induction on $n$. As we have $S P(3,2,\{1,2\},\{2\})=-36, S P(3,2,\{2\}, \emptyset)=36$. Lemma 5 holds for $n=3$. Suppose that Lemma 5 is proved for $n=N-1, N \geq 3$. First, we shall 
prove Lemma 5 for $n=N, \lambda \neq N-1$. Let $I P(N, \lambda, I, J)$ be the function defined by

(49)

$$
I P(N, \lambda, I, J)=\left\{\begin{aligned}
\prod_{\nu \in I+\{\lambda-1\}+\{\lambda\}}\left(\frac{N-1+\nu}{N-1-\nu}\right) \prod_{\nu \in J+\{\lambda\}}\left(\frac{N-1+\nu}{N-1-\nu}\right), & \text { if } N-1 \notin I, N-1 \notin J, \\
-\prod_{\nu \in I^{\prime}+\{\lambda\}}\left(\frac{N-1+\nu}{N-1-\nu}\right) \prod_{\nu \in J+\{\lambda\}}\left(\frac{N-1+\nu}{N-1-\nu}\right), & \text { if } N-1 \in I, N-1 \notin J, \\
-\prod_{\nu \in I+\{\lambda-1\}+\{\lambda\}}\left(\frac{N-1+\nu}{N-1-\nu}\right) \prod_{\nu \in J^{\prime}}\left(\frac{N-1+\nu}{N-1-\nu}\right), & \text { if } N-1 \notin I, N-1 \in J, \\
\prod_{\nu \in I^{\prime}+\{\lambda\}}\left(\frac{N-1+\nu}{N-1-\nu}\right) \prod_{\nu \in J^{\prime}}\left(\frac{N-1+\nu}{N-1-\nu}\right), & \text { if } N-1 \in I, N-1 \in J .
\end{aligned}\right.
$$

By Propositions 1, 2 and Definitions 1, 2, we have

$$
\begin{aligned}
& S P(N, \lambda, I+\{\lambda-1\}+\{\lambda\}, J+\{\lambda\}) \\
& \quad=S P(N-1, \lambda, I+\{\lambda-1\}+\{\lambda\}, J+\{\lambda\}) I P(N, \lambda, I, J) \\
& S P\left(N, 1, I^{\prime}+\{\lambda\}, J^{\prime}\right) \\
& \quad=S P\left(N-1,1, I^{\prime}+\{\lambda\}-\{N-1\}, J^{\prime}-\{N-1\}\right) I P(N, \lambda, I, J) .
\end{aligned}
$$

By the induction hypothesis, we have

$$
\begin{aligned}
& S P(N, \lambda, I+\{\lambda-1\}+\{\lambda\}, J+\{\lambda\})+S P\left(N, \lambda, I^{\prime}+\{\lambda\}, J^{\prime}\right) \\
& =I P(N, \lambda, I, J) \\
& \times\{S P(N-1, \lambda, I+\{\lambda\}, J+\{\lambda\}) \\
& \left.+S P\left(N-1, \lambda, I^{\prime}+\{\lambda-1\}+\{\lambda\}-\{N-1\}, J^{\prime}-\{N-1\}\right)\right\} \\
& =0 \text {. }
\end{aligned}
$$

Hence, we have verified Lemma 5 for $n=N, \lambda \neq N-1$. Now, we shall prove Lemma 5 for $n=N, \lambda=N-1$. By Propositions 1, 2 and Definitions 1, 2, we have

$$
\begin{aligned}
& S P(N, N-1, I+\{N-2\}+\{N-1\}, J+\{N-1\}) \\
& \quad=-2(N-1)(2 N-3) \prod_{\nu \in I}\left(\frac{N-1+\nu}{N-1-\nu}\right) \prod_{\nu \in J}\left(\frac{N-2-\nu}{N-2+\nu}\right)
\end{aligned}
$$




$$
\times \mu_{N}(I+\{N-2\}+\{N-1\}) \mu_{N}(J+\{N-1\}) .
$$

We shall prove

$$
\begin{aligned}
\mu_{N}\left(I^{\prime}+\{N-1\}\right)= & \prod_{\nu \in I+\{N-2\}}\left(\frac{N-1+\nu}{N-1-\nu}\right) \prod_{\nu \in I^{\prime}}\left(\frac{N-1-\nu}{N-1+\nu}\right) \\
& \times \mu_{N}(I+\{N-2\}+\{N-1\}) .
\end{aligned}
$$

It follows from Proposition 2 that

$$
\mu\left(I^{\prime}+\{N-1\}\right)=\mu(I+\{N-2\})
$$

by Proposition 1 for $n=N-1$,

$$
=\prod_{\nu \in I+\{N-2\}}\left(\frac{N-1+\nu}{N-1-\nu}\right) \mu_{N-1}(I+\{N-2\})
$$

by Proposition 2,

$$
=\prod_{\nu \in I+\{N-2\}}\left(\frac{N-1+\nu}{N-1-\nu}\right) \mu_{N-1}\left(I^{\prime}\right)
$$

by Proposition 1 for $n=N-1$,

$$
=\prod_{\nu \in I+\{N-2\}}\left(\frac{N-1+\nu}{N-1-\nu}\right) \prod_{\nu \in I^{\prime}}\left(\frac{N-1-\nu}{N-1+\nu}\right) \mu_{N}\left(I^{\prime}\right)
$$

by Proposition 2,

$$
=\prod_{\nu \in I+\{N-2\}}\left(\frac{N-1+\nu}{N-1-\nu}\right) \prod_{\nu \in I^{\prime}}\left(\frac{N-1-\nu}{N-1+\nu}\right) \mu_{N}(I+\{N-2\}+\{N-1\}) .
$$

We hence have the identity (54). So it follows from the identities (54), (44) and Definitions 1, 2,

$$
\begin{aligned}
S P(N, & \left.N-1, I^{\prime}+\{N-1\}, J^{\prime}\right) \\
= & 2(N-1)(2 N-3) \prod_{\nu \in I}\left(\frac{N-1+\nu}{N-1-\nu}\right) \prod_{\nu \in J}\left(\frac{N-2-\nu}{N-2+\nu}\right) \\
& \times \mu_{N}(I+\{N-2\}+\{N-1\}) \mu_{N}(J+\{N-1\}) .
\end{aligned}
$$


Combining the identity (56) with the identity (53), we have

$$
\begin{gathered}
S P(N, N-1, I+\{N-2\}+\{N-1\}, J+\{N-1\}) \\
\quad+S P\left(N, N-1, I^{\prime}+\{N-1\}, J^{\prime}\right)=0 .
\end{gathered}
$$

Lemma 5 holds for $n=N, \lambda=N-1$. With these result, we have verified Lemma 5 for $n=N$ and hence obtain Lemma 5 by mathematical induction.

To prove Theorem 1 , we use the following

Proposition 4. For an integer $n \geq 3$, an integer $2 \leq \lambda \leq n-1$ and subsets $I, J \subset[n-1]-\{\lambda\}-\{\lambda-1\}$, we have

$$
\begin{aligned}
& S P(n, \lambda, I+\{\lambda-1\}+\{\lambda\}, J+\{\lambda-1\}+\{\lambda\})=0, \\
& S P(n, \lambda, I+\{\lambda\}, J+\{\lambda-1\})=0, \\
& S P(n, \lambda, I+\{\lambda-1\}+\{\lambda\}, J+\{\lambda-1\})=0, \\
& S P(n, \lambda, I+\{\lambda-1\}, J+\{\lambda\})=0, \\
& S P(n, \lambda, I+\{\lambda-1\}, J+\{\lambda-1\}+\{\lambda\})=0 .
\end{aligned}
$$

For an integer $n \geq 2$, and subsets $I, I^{\prime}, J, J^{\prime}$ such that $I+I^{\prime}=[n-1]-\{1\}$, $J+J^{\prime}=[n-1]-\{1\}$, we have

$$
\begin{aligned}
& S P(n, 1, I+\{1\}, J+\{1\})+S P\left(n, 1, I^{\prime}, J^{\prime}+\{1\}\right) \\
& \quad+S P\left(n, 1, I^{\prime}+\{1\}, J^{\prime}\right)=0 .
\end{aligned}
$$

For an integer $n \geq 3$, an integer $2 \leq \lambda \leq n-1$, and subsets $I, I^{\prime}, J, J^{\prime}$ such that $I+I^{\prime}=[n-1]-\{\lambda\}-\{\lambda-1\}, J+J^{\prime}=[n-1]-\{\lambda\}-\{\lambda-1\}$, we have

$$
\begin{aligned}
& \quad S P(n, \lambda, I+\{\lambda\}, J+\{\lambda\})+S P\left(n, \lambda, I^{\prime}, J^{\prime}+\{\lambda-1\}+\{\lambda\}\right) \\
& \quad+S P\left(n, \lambda, I^{\prime}+\{\lambda-1\}+\{\lambda\}, J^{\prime}\right)=0 \\
& \quad S P(n, \lambda, I+\{\lambda-1\}+\{\lambda\}, J+\{\lambda\})+S P\left(n, \lambda, I^{\prime}+\{\lambda\}, J^{\prime}\right)=0 \\
& S P(n, \lambda, I+\{\lambda\}, J+\{\lambda-1\}+\{\lambda\})+S P\left(n, \lambda, I^{\prime}, J^{\prime}+\{\lambda\}\right)=0 .
\end{aligned}
$$

Proof. From Lemma 2, we have the identities (58), (59), (60), (61) and (62). We have

$$
\begin{aligned}
& S P(n, 1, I+\{1\}, J+\{1\}) \\
& \quad=T P(n, 1, I+\{1\} ; J+\{1\})+T P(n, 1, J+\{1\} ; I+\{1\})
\end{aligned}
$$


for an integer $n \geq 2$ and $I, J \subset[n-1]-\{1\}$. By Lemma 3, we hence have the identity (63). We have

$$
\begin{aligned}
& S P(n, \lambda, I+\{\lambda\}, J+\{\lambda\}) \\
& \quad=T P(n, \lambda, I+\{\lambda\} ; J+\{\lambda\})+T P(n, \lambda, J+\{\lambda\} ; I+\{\lambda\})
\end{aligned}
$$

for an integer $n \geq 2$ and $I, J \subset[n-1]-\{1\}$. By Lemma 4, we hence have the identity (64). We proved already the identity (65) in Lemma 5 . We note that the identity (66) is equivalent to the identity (65).

\section{§2. Proof of Theorem 1}

We put $v=e^{x}+e^{-x}$ and define the operators $l_{x}(f)$ and $l_{x}(f, g)$ by

$$
\begin{gathered}
l_{x}(f)=f \frac{d^{2} f}{d x^{2}}-\left(\frac{d f}{d x}\right)^{2} \\
l_{x}(f, g)=f \frac{d^{2} g}{d x^{2}}-2 \frac{d f}{d x} \frac{d g}{d x}+g \frac{d^{2} f}{d x^{2}}
\end{gathered}
$$

Let $c$ be a constant, $f, g$ be functions of variable $x$ and $n, m$ be non-negative integers. Then we have

$$
\begin{aligned}
& l_{x}(c f)=c^{2} l_{x}(f), \quad l_{x}(f, g)=l_{x}(g, f), \quad l_{x}(c f, g)=c l_{x}(f, g), \\
& l_{x}(f g)=f^{2} l_{x}(g)+g^{2} l_{x}(f), \quad l_{x}(f+g)=l_{x}(f)+l_{x}(g)+l_{x}(f, g) .
\end{aligned}
$$

By $z=(2-v) / 4, w=(2+v) / 4, v=e^{x}+e^{-x}$, we have

$$
z w l_{x}\left(z^{n} w^{m}\right)=((n / 2) w+(m / 2) z) z^{2 n} w^{2 m}
$$

and for $n_{1}+m_{1}=n_{2}+m_{2}$

$$
\begin{aligned}
z w l_{x} & \left(z^{n_{1}} w^{m_{1}}, z^{n_{2}} w^{m_{2}}\right) \\
= & {\left[\left\{(1 / 2)\left(n_{1}+n_{2}\right)-\left(n_{1}-n_{2}\right)^{2}\right\} w\right.} \\
& \left.+\left\{(1 / 2)\left(m_{1}+m_{2}\right)-\left(m_{1}-m_{2}\right)^{2}\right\} z\right] z^{n_{1}+n_{2}} w^{m_{1}+m_{2}} .
\end{aligned}
$$

We now define $U_{n}$ for $n \geq 2$ by

$$
U_{n}=\sum_{I \subset[n-1]} \mu_{n}(I) c_{I} d_{[n-1]-I} z^{\|I\|} w^{\|[n-1]-I\|},
$$


and denote $U_{0}=U_{1}=1$. We prove that, for a positive integer $n, U_{n}$ satisfies the following recurrence relation

$$
U_{n-1} U_{n+1}=\left(\overline{c_{n}} z+\overline{d_{n}} w\right) U_{n}^{2}-16 z w l_{x}\left(U_{n}\right)
$$

which is equivalent to the fact that $T_{n}$ presented by Theorem 1 satisfies the recurrence relation (1). As we have $U_{0}=U_{1}=1, U_{2}=c_{1} z+d_{1} w$. The recurrence relation (74) holds for $n=1$. We have to prove the recurrence relation (74) for $n \geq 2$. We then have for $n \geq 2$

$$
\begin{aligned}
& \left.\overline{\left(c_{n}\right.} z+\overline{d_{n}} w\right) U_{n}^{2}-16 z w l_{x}\left(U_{n}\right) \\
& =\sum_{I, J \subset[n-1]} P(I, J) c_{I} d_{[n-1]-I} c_{J} d_{[n-1]-J} z^{\|I\|+\|J\|} w^{\|2[n-1]\|-\|I\|-\|J\|+1} \\
& \quad+\sum_{I, J \subset[n-1]} P^{*}(I, J) c_{[n-1]-I} d_{I} c_{[n-1]-J} d_{J} z^{\|2[n-1]\|-\|I\|-\|J\|+1} w^{\|I\|+\|J\|}
\end{aligned}
$$

where $P(I, J), P^{*}(I, J)$ for an integer $n \geq 2$ and subsets $I, J \subset[n-1]$ are defined by

$$
\begin{gathered}
P(I, J)=\mu_{n}(I) \mu_{n}(J)\left(\overline{d_{n}}-4(\|I\|+\|J\|)+8(\|I\|-\|J\|)^{2}\right), \\
P^{*}(I, J)=\mu_{n}(I) \mu_{n}(J)\left(\overline{c_{n}}-4(\|I\|+\|J\|)+8(\|I\|-\|J\|)^{2}\right) .
\end{gathered}
$$

Using $b_{\lambda}^{I, J}$ and Lemma 1 , we deform the polynomial $P(I, J)$ by

$$
\begin{aligned}
P(I, J)= & \mu_{n}(I) \mu_{n}(J)\left(\overline{d_{n}}-4(\|I\|+\|J\|)+8(\|I\|-\|J\|)^{2}\right) \\
= & \mu_{n}(I) \mu_{n}(J)\left(\overline{d_{n}}+2 \sum_{\lambda \in I \cup J} b_{\lambda}^{I, J}\right) \\
= & \mu_{n}(I) \mu_{n}(J) \overline{d_{n}}\left(1+\sum_{\lambda \in I \cup J} \frac{b_{\lambda}^{I, J}}{2(n-\lambda)(n-1+\lambda)}\right) \\
& -\sum_{\lambda \in I \cup J} \frac{b_{\lambda}^{I, J} \mu_{n}(I) \mu_{n}(J)\left(\overline{d_{n}}-4(n-\lambda)(n-1+\lambda)\right)}{2(n-\lambda)(n-1+\lambda)} .
\end{aligned}
$$

Combining the definitions of $F P(I, J)$ and $S P(n, \lambda, I, J)$ with the identity (78), we have by $\left(\overline{d_{n}}-4(n-\lambda)(n-1+\lambda)\right)=\overline{d_{\lambda}}$

$$
P(I, J)=F P(I, J) \overline{d_{n}}-\sum_{\lambda \in I \cup J} \frac{S P(n, \lambda, I, J) \overline{d_{\lambda}}}{2(n-\lambda)(n-1+\lambda)} .
$$


Similarly, we have

$$
P^{*}(I, J)=F P(I, J) \overline{c_{n}}-\sum_{\lambda \in I \cup J} \frac{S P(n, \lambda, I, J) \overline{c_{\lambda}}}{2(n-\lambda)(n-1+\lambda)} .
$$

For an integer $n \geq 2$, subsets $I, J \subset[n-1]$ and an integer $\lambda \in I \cup J$, we define

$$
\begin{aligned}
& E(n, \lambda, I, J)=\overline{d_{\lambda}} c_{I} d_{[n-1]-I} c_{J} d_{[n-1]-J} z^{\|I\|+\|J\|} w^{\|2[n-1]\|-\|I\|-\|J\|+1} \\
& E^{*}(n, \lambda, I, J)=\overline{c_{\lambda}} c_{[n-1]-I} d_{I} c_{[n-1]-J} d_{J} z^{\|2[n-1]\|-\|I\|-\|J\|+1} w^{\|I\|+\|J\|}
\end{aligned}
$$

We then have the following

Lemma 6. For an integer $n \geq 2$, and subsets $I, I^{\prime}, J, J^{\prime}$ such that $I+I^{\prime}=$ $[n-1]-\{1\}, J+J^{\prime}=[n-1]-\{1\}$, we have

$$
E(n, 1, I+\{1\}, J+\{1\})=E^{*}\left(n, 1, I^{\prime}, J^{\prime}+\{1\}\right)=E^{*}\left(n, 1, I^{\prime}+\{1\}, J^{\prime}\right) .
$$

For an integer $n \geq 3$, an integer $2 \leq \lambda \leq n-1$, and subsets $I, I^{\prime}, J, J^{\prime}$ such that $I+I^{\prime}=[n-1]-\{\lambda\}-\{\lambda-1\}, J+J^{\prime}=[n-1]-\{\lambda\}-\{\lambda-1\}$, we have

$$
\begin{aligned}
& E(n, \lambda, I+\{\lambda\}, J+\{\lambda\})=E^{*}\left(n, \lambda, I^{\prime}, J^{\prime}+\{\lambda-1\}+\{\lambda\}\right) \\
& =E^{*}\left(n, \lambda, I^{\prime}+\{\lambda-1\}+\{\lambda\}, J^{\prime}\right) \\
& E(n, \lambda, I+\{\lambda-1\}+\{\lambda\}, J+\{\lambda\})=E^{*}\left(n, \lambda, I^{\prime}+\{\lambda\}, J^{\prime}\right) \\
& E(n, \lambda, I+\{\lambda\}, J+\{\lambda-1\}+\{\lambda\})=E^{*}\left(n, \lambda, I^{\prime}, J^{\prime}+\{\lambda\}\right) .
\end{aligned}
$$

We note the following

Lemma 7. For an integer $n \geq 2$ and subsets $I, J \subset[n-1]$ such that $n-1 \notin I$, we have

$$
\begin{aligned}
E(n, n, I, J) & =c_{I} d_{[n-2]-I} z^{\|I\|} w^{\|[n-2]-I\|} c_{J} d_{[n]-J} z^{\|J\|} w^{\|[n]-J\|} \\
E^{*}(n, n, I, J) & =c_{[n-2]-I} d_{I} z^{\|[n-2]-I\|} w^{\|I\| \mid} c_{[n]-J} d_{J} z^{\|[n]-J\|} w^{\|J\|}
\end{aligned}
$$

From Proposition 4 and Lemma 6, we have

$$
\begin{aligned}
& \sum_{I, J \subset[n-1]} \sum_{\lambda \in I \cup J} \frac{S P(n, \lambda, I, J) E(n, \lambda, I, J)}{2(n-\lambda)(n-1+\lambda)} \\
& +\sum_{I, J \subset[n-1]} \sum_{\lambda \in I \cup J} \frac{S P(n, \lambda, I, J) E^{*}(n, \lambda, I, J)}{2(n-\lambda)(n-1+\lambda)}=0 .
\end{aligned}
$$


Combining the identities (75), (79), (80) with the identity (89) and combining Proposition 3 with Lemma 7 , we have for an integer $n \geq 2$

$$
\begin{aligned}
& \left.\overline{\left(c_{n}\right.} z+\overline{d_{n}} w\right) U_{n}^{2}-16 z w l_{x}\left(U_{n}\right) \\
& =\sum_{I, J \subset[n-1]} F P(I, J) E(n, n, I, J)+\sum_{I, J \subset[n-1]} F P(I, J) E^{*}(n, n, I, J) \\
& =U_{n-1} U_{n+1} .
\end{aligned}
$$

Consequently we have verified that $U_{n}$ satisfy the recurrence relation (74). So, $T_{n}$ given by Theorem 1 satisfies the recurrence relation (1). Since $T_{n}$ is uniquely determined by the recurrence relation (1), Theorem 1 holds.

\section{References}

[1] H. Umemura, Special polynomials associated with the Painlevé equation I, Proceedings of workshop on the Painlevé equations (edited by Winternitz), Montreal, (1996).

[2] M. Noumi, K. Okamoto, S. Okada and H. Umemura, Special polynomials associated with the Painlevé equation II, Proceeding of Taniguchi Symp., 1997; Integrable systems and algebraic geometry, World Scientific, 1998.

KuMAMOTO HidA 4-3-87

KUMAMOTO 861-5514, JAPAN

E-mail: tane@rc4.so-net.ne.jp 